\title{
Cosmic ray contribution in extragalactic background gamma-ray emission at an energy of $0.1 \mathrm{TeV}$
}

\author{
Anna Uryson ${ }^{\mathrm{a}}$ \\ Lebedev Physical Institute RAS, 119991 Moscow, Russia
}

\begin{abstract}
We estimate the intensity of gamma-quanta at the energy $0.1 \mathrm{TeV}$ that is generated in extragalactic space in interactions of ultra-high energy cosmic rays with the cosmic background emission. Energy of $0.1 \mathrm{TeV}$ is chosen because the Universe is mostly transparent for these quanta. In the paper three types of cosmic ray sources are analyzed: objects with red shifts up to $z=1.1$ having monoenergetic particle spectra, $E=10^{21} \mathrm{eV}$; the same objects with exponential particle spectra; objects with red shifts $0<z \leq 0.0092$ i.e. located at distances less than $\approx 50 \mathrm{Mpc}$, also with exponential particle spectra. It is found that the cosmic ray contribution in extragalactic background emission at $0.1 \mathrm{TeV}$ ranges from $f \ll 10^{-4}$ to $f \approx 0.1$ depending on the source characteristics. Thus the cosmic ray contribution in extragalactic background emission can be used for studying cosmic ray sources.
\end{abstract}

\section{Introduction}

The main problem of ultra-high energy (UHE) cosmic rays (CRs) is where they come from. Point UHECR sources seem to be appropriate but they are not discovered.

Information about UHECR origin is usually obtained from the particle energy spectrum. In space, particles lose energy in interactions with the cosmic microwave background. This results in a lack of particles at $E>10^{20} \mathrm{eV}$ at the Earth and in spectrum suppression (GZK-effect: [1,2]) if UHECRs come from distances of more than $\approx 100 \mathrm{Mpc}$. If particles arrive from nearer distances the UHECR spectrum has no GZK-suppression. Experimental data obtained with CR giant arrays - Pierre Auger Observatory and Telescope Array - indicate that the UHECR energy spectrum is suppressed. However, there may be another reason for spectrum suppression besides the GZK-effect: particles are accelerated to the maximum energy of approximately $10^{21} \mathrm{eV}$ due to conditions in possible UHECR sources [3,4]. As sources are still unknown, the acceleration energy limit cannot be ruled out. So the reason for the spectrum suppression is not clear.

In addition to GZK-suppression there is another effect of UHECR interactions with the cosmic microwave background. Particles initiate electromagnetic cascades in extragalactic space $[5,6]$. We calculate the intensity of cascade quanta at the energy $0.1 \mathrm{TeV}$ and find that it depends on the source characteristics: remoteness and initial CR energy spectra. We chose this energy because the Universe is almost transparent for such quanta [7], so their intensity is maximal.

\section{Extragalactic cascades}

In extragalactic space UHECRs interact with the microwave background: $\quad p+\gamma_{\text {rel }} \longrightarrow p+\pi^{0}$,

a e-mail: uryson@sci.lebedev.ru $p+\gamma_{r e l} \longrightarrow n+\pi^{+}$. The cross section of reactions depends on the energies of the interacting particles. It has a peak of approximately $0.44 \mathrm{mb}$ at photon energy $\epsilon^{*} \approx 340 \mathrm{GeV}$ in the proton rest system [8]. Thus the $\mathrm{CR}$ free path depends on the particle energy. For CRs at energies of about $4 \cdot 10^{19} \mathrm{eV}$ their paths are $\sim 100 \mathrm{Mpc}$, whereas particles at energies approximately $10^{20} \mathrm{eV}$ have paths of only $\sim 10 \mathrm{Mpc}[9]$. The energy transfer to pions is determined by the inelasticity of the interaction that is also given in [9].

Particles cascading is described in $[5,6]$. Generated pions decay via reactions $\pi^{0} \longrightarrow 2 \gamma, \pi^{+} \longrightarrow \mu^{+}+$ $v, \mu^{+} \longrightarrow e^{+}+v+\bar{v}$, producing electrons and quanta which give rise to cascades.

The cascade develops through electron and quanta reactions with background emission including not only relic emission but also radio photons [10]. These reactions are pair production (PP) $\gamma+\gamma_{b} \longrightarrow e^{+}+e^{-}$and inverse Compton scattering (ICS) $e+\gamma_{b} \longrightarrow e^{\prime}+\gamma^{\prime}$ (here index $b$ denotes background photons, index ' denotes scattered particles).

PP takes place provided that the photon energy is above the threshold energy

$$
E_{t}=\left(m c^{2}\right)^{2} / \epsilon_{b}
$$

where $m c^{2}$ is the electron mass, $m c^{2}=0.511 \mathrm{MeV}$, and $\epsilon_{b}$ is the energy of the background photon.

Cross sections of the PP and ICS are known [11]. For $\mathrm{PP}$ it is

$$
\begin{aligned}
\sigma_{\gamma \gamma} & \approx 3 / 8 \sigma_{T} a^{2} /\left\{\left[2+2 a^{2}-a^{4}\right] \ln \left(a^{-1}+\left(a^{-2}-1\right)^{1 / 2}\right)\right. \\
& \left.-\left(1-a^{2}\right)^{1 / 2}\left(1+a^{2}\right)\right\},
\end{aligned}
$$

where $a=m c^{2} / E_{e c}, E_{e c}$ is the photon energy in the centre-of-mass system,

$$
E_{e c}=\left[E_{e} \epsilon_{b}(1-\cos \psi)\right]^{1 / 2},
$$

(C) The Authors, published by EDP Sciences. This is an Open Access article distributed under the terms of the Creative Commons Attribution License 4.0 (http://creativecommons.org/licenses/by/4.0/). 
and $\psi$ is the angle between photon impulses in the laboratory system. The cross section of the ICS at $E_{e}>E_{t}$ equals

$$
\sigma_{I C} \approx 3 / 8 \sigma_{T}\left(m c^{2}\right)^{2} /\left(E_{e} \epsilon_{b}\right)\left\{\ln \left[2 E_{e} \epsilon_{b}\left(m c^{2}\right)^{2}\right]+0.5\right\}
$$

and equals the Thomson cross section, $\sigma_{T} \approx 6.65$. $10^{-25} \mathrm{~cm}^{2}$, at $E_{e}<E_{t}$.

For electrons or quanta at lower energies, $E_{e}<E_{t}$, there is no PP, and comparatively soft quanta are produced in ICS, at mean energies

$$
E_{\gamma}=4 / 3 \epsilon_{b}\left(E_{e} / m c^{2}\right)^{2}
$$

In this case the electron loses energy fairly slowly.

Minor interactions and the influence of magnetic fields in extragalactic space are discussed in [12].

Cosmic particles move in the expanding Universe and lose energy adiabatically. Passing the distance from a point with red shift $z_{i}$ to a point with red shift $z_{i+1}$ the particle energy decrease $\left(\Delta E_{a d}\right)$ equals:

$$
\left(\Delta E_{a d}\right)=E\left(z_{i+1}-z_{i}\right) /\left(1+z_{i}\right) .
$$

\section{The model}

Model assumptions concern three points: CR sources, background emission in extragalactic space, and the extragalactic magnetic field.

We assume that $\mathrm{CR}$ sources are point. There is no contradiction with results [13].

Next we assume that UHECR sources are active galactic nuclei: Blue Lacertae objects (Bl Lacs) that are powerful and remote at distances of hundreds Mpc (with red shifts up to $z=1.1$ ), and Seyfert nuclei located in the radius of about $50 \mathrm{Mpc}$ (with red shifts $0<$ $z \leq 0.0092$ ) which are comparatively low-power. These possible UHECR sources were analyzed in [14-17].

We obtained spatial distributions of sources using data on object red shifts from the catalogue [18]. The distributions are presented in [19].

We suppose that at $z>0.1$ distance $r$ is related to red shift $z$ as

$$
r=2 / 3 c H^{-1}\left(1-(1+z)^{-3 / 2}\right)(\mathrm{Mpc})
$$

(in accordance with the Einstein-de Sitter model with the parameter $\Omega=1$ ), and as

$$
r=c z / H(\mathrm{Mpc})
$$

at lower $z$.

We assume a simple case, when CRs are accelerated in sources by either electrical fields, or by shock waves. In the first case the initial CR spectrum is monoenergetic, in the second it is exponential [19]. To describe UHECR energy spectra measured at the Earth the particle spectrum in $\mathrm{Bl}$ Lacs is either monoenergetic with energy $E=10^{21} \mathrm{eV}$ or exponential with the index in the range 2-3; in Seyfert nuclei the CR spectrum is exponential with the index in the range 2-3. Particles in sources are accelerated to the energy not higher than $10^{21} \mathrm{eV}[3,4]$.

Hereinafter B1 Lacs are called remote sources, Seyferts are called near ones.

Thus in total we consider three types of UHECR sources: remote ones with a particle monoenergy spectrum, remote sources with the $\mathrm{CR}$ exponential spectrum, and near ones with the particle exponential spectrum.

We assume also that CRs consist of protons.

In the model, background emission is treated in the following way. The microwave background emission has Planck distribution in energy with a mean value $\epsilon_{r}=6.7 \cdot 10^{-4} \mathrm{eV}$, the mean photon density is $n_{r}=400 \mathrm{~cm}^{-3}$. The high-energy "tail" consists of photons at the energy $\epsilon_{t}=1 \cdot 10^{-3} \mathrm{eV}$, their mean density is $n_{t}=42 \mathrm{~cm}^{-3}$. The threshold energy (1) of PP and ICS equals $E_{t} \approx 10^{14} \mathrm{eV}$. Microwave background anisotropy has no influence on the cascade and is not considered.

The spectrum of the extragalactic background radiation was obtained experimentally in [20] and later theoretically in [21] allowing for the evolution of radio sources. We use results [21]: the radio background exists at the energy $\epsilon_{\text {rad }} \approx 4 \cdot 10^{-10} \mathrm{eV}$, so the threshold energy (1) equals $E_{t} \approx 6 \cdot 10^{20} \mathrm{eV}$.

We neglect electron losses due to synchrotron radiation in extragalactic magnetic fields.

\section{Calculations}

First we determine the number of gamma-quanta at the energy $E_{\gamma} \approx 10^{14} \mathrm{eV}$ produced by a $\mathrm{CR}$ proton propagating from the source to the Galaxy by the following scheme.

Distances from UHECR sources are obtained in accordance with their spatial distribution: a source red shift, $z_{s}$, is generated by Monte-Carlo techniques, and the distance to the source is calculated. The proton mean free path in interactions with background emission being $<L>=1 /\left(n_{r e l} \sigma_{\gamma}\right)$, the proton free path $L$ is generated by the Monte-Carlo method.

At the point of interaction proton energy losses $\Delta\left(E_{a d}\right)$ are calculated due to the expansion of the Universe. Then the energy of the CR proton after interaction is obtained. Both the cross-section of interaction and energy transfer are calculated using their dependences on $\epsilon^{*}$ [9]. The procedure is repeated until the proton reaches the Galaxy either its energy decreases to $E<4 \cdot 10^{19} \mathrm{eV}$ (because protons at this energy have paths of several hundred Mpc).

In $\pi^{0}$-decays quanta are produced at energy $E_{\gamma}=$ $0.5 E_{\pi^{0}}$. In PP according to Eqs. (1-3), the angle $\psi$ between impulses of a cascade photon and a background one is generated in the observer's frame of reference, then the photon energy $E_{e c}$ in the centre of mass system is calculated. Next we obtain values of the cross section, $\sigma_{\gamma \gamma}$, and the free path of a cascade quanta $\lambda_{\gamma \gamma}=1 /\left(n_{b} \sigma_{\gamma \gamma}\right)$. We chose between a radio photon and a relic one that photon whose path is shorter. Then the threshold energy $E_{t}$ is derived according to Eq. (1). We suppose that one of the particles in the electron-positron pair has the energy $E_{t}$ and the other one is at the energy $\left(E_{\gamma}-E_{t}\right)$. The ICS is considered in the same way using Eqs. $(4,5)$. The electron is treated until its energy decreases to $10^{14} \mathrm{eV}$ (as at lower energies it loses energy fairly slowly scattering soft quanta).

Using this scheme we obtain the total number of gamma-quanta, $N_{\gamma}$, at energy $E \approx 10^{14} \mathrm{eV}$ produced by one proton passing from a source to the Galaxy. The procedure is repeated for 1000 sources emitting one 
proton. Then the cascade-mean number of gamma-quanta at the energy $E \approx 10^{14} \mathrm{eV}$ is derived.

A gamma-quantum at $E_{\gamma} \approx 10^{14} \mathrm{eV}$ interacts with extragalactic background light (EBL) until its energy decreases to $E_{\gamma 1} \approx 0.1 \mathrm{TeV}$. (The Universe is transparent for gamma-ray emission at this energy [7].) We suppose that in interactions with EBL a quantum at $E_{\gamma} \approx 100 \mathrm{TeV}$ produces $M_{\gamma}=10^{3}$ quanta at the energy $E_{\gamma 1}=0.1 \mathrm{TeV}$, $M_{\gamma}=E_{\gamma} / E_{\gamma 1}$. As a result a CR proton propagating from the source to the Galaxy produces $N_{\gamma} M_{\gamma}$ gamma-quanta at the energy $E_{\gamma 1} \approx 0.1 \mathrm{TeV}$.

\section{Results}

In the model of remote sources with the $\mathrm{CR}$ monoenergetic initial spectrum, $E=10^{21} \mathrm{eV}$, protons on their way to the Galaxy give rise to $N_{\gamma} \approx 10^{6}$ gamma-quanta at the energy $E_{\gamma} \approx 100 \mathrm{TeV}$, which in turn produce $M_{\gamma}=10^{9}$ quanta at $E_{\gamma 1}=0.1 \mathrm{TeV}$.

Sources with a CR exponential initial energy spectra result in smaller values of $N_{\gamma}$. Protons from remote sources initiate $N_{\gamma} \approx 1.5 \cdot 10^{3}$ quanta at $E_{\gamma} \approx 100 \mathrm{TeV}$ along their way to the Galaxy, and produce $M_{\gamma}=1.5 \cdot 10^{6}$ quanta at $E_{\gamma 1}=0.1 \mathrm{TeV}$. Near sources (which are Seyfert nuclei) result in $N_{\gamma} \approx 0, M_{\gamma} \approx 0$. These values of $N_{\gamma}$ do not depend on the exponential index of the initial energy spectrum.

The intensity of gamma-ray emission produced in extragalactic cascades $I_{\gamma}(\geq 0.1 \mathrm{TeV})$ is estimated in the following way.

$$
I_{\gamma}(\geq 0.1 \mathrm{TeV})=M_{\gamma} I_{C R}\left(E>4 \cdot 10^{19} \mathrm{eV}\right) .
$$

Approximations of the CR integral intensity are [22]:

$$
I_{C R}(>E)=1 \cdot(E(\mathrm{GeV}))^{-1.7}\left(\mathrm{~cm}^{2} \mathrm{~s} \mathrm{sr}\right)^{-1}
$$

at $10 \mathrm{GeV}<E<3 \cdot 10^{6} \mathrm{GeV}$;

$$
I_{C R}(>E)=3 \cdot 10^{-10}\left(E(\mathrm{GeV}) / 10^{6}\right)^{-2.1}\left(\mathrm{~cm}^{2} \mathrm{~s} \mathrm{sr}\right)^{-1}
$$

at $E>3 \cdot 10^{6} \mathrm{GeV}$. Using these approximations we get

$$
I_{C R}\left(E>4 \cdot 10^{19} \mathrm{eV}\right) \approx 6 \cdot 10^{-20}\left(\mathrm{~cm}^{2} \mathrm{~s} \mathrm{sr}\right)^{-1} .
$$

In our models the intensity of cascade gamma-ray emission is following remote and powerful sources with initial monoenergetic energy spectra, $E=10^{21} \mathrm{eV}$ :

$$
I_{\gamma}(E \geq 0.1 \mathrm{TeV}) \approx 6 \cdot 10^{-11}\left(\mathrm{~cm}^{2} \mathrm{~s} \mathrm{sr}\right)^{-1}
$$

remote sources with initial exponential energy spectra:

$$
I_{\gamma}(E \geq 0.1 \mathrm{TeV}) \approx 6 \cdot 10^{-14}\left(\mathrm{~cm}^{2} \mathrm{~s} \mathrm{sr}\right)^{-1},
$$

near sources with the initial exponential energy spectra:

$$
I_{\gamma}(E \geq 0.1 \mathrm{TeV}) \ll 6 \cdot 10^{-14}\left(\mathrm{~cm}^{2} \mathrm{~s} \mathrm{sr}\right)^{-1} .
$$

Fermi LAT gives data on the intensity of extragalactic background gamma-ray emission [23]:

$$
I_{E G B}(>0.1 \mathrm{TeV}) \approx 7 \cdot 10^{-10}\left(\mathrm{~cm}^{2} \mathrm{~s} \mathrm{sr}\right)^{-1} .
$$

Thus the fraction of cascade quanta in the intensity of extragalactic background gamma-ray emission depends on CR source parameters. At the energy $E_{\gamma 1} \approx 0.1 \mathrm{TeV}$ the fraction ranges from $f \ll 10^{-4}$ to $f \approx 0.1$.

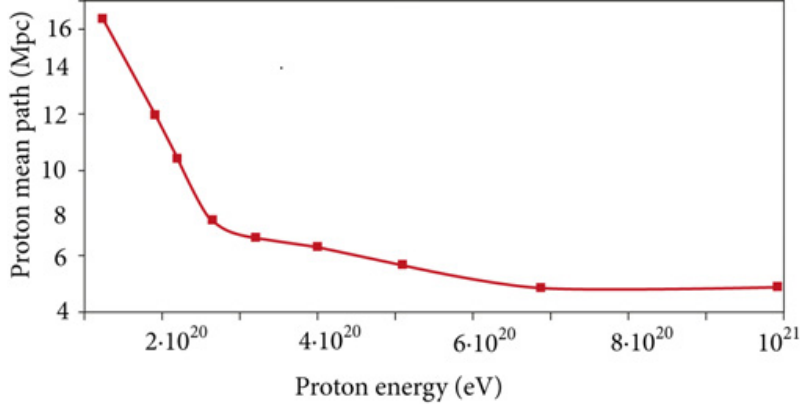

Figure 1. Proton mean path vs. proton energy in extragalactic space.

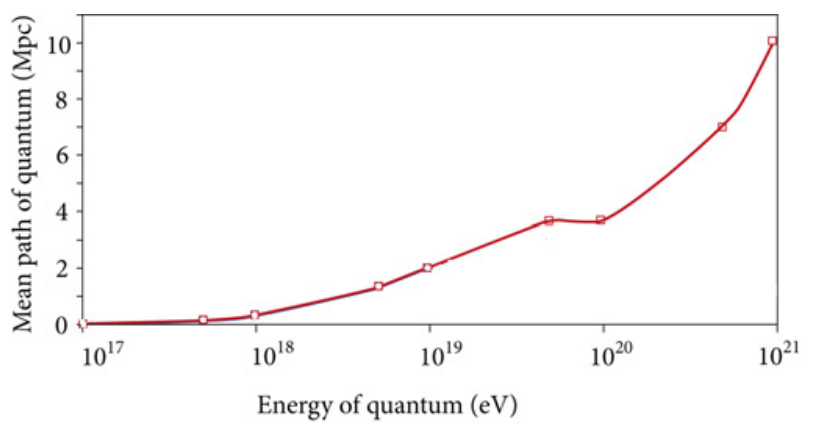

Figure 2. Quantum mean path vs. its energy in PP in extragalactic space.

\section{Discussion}

The variety in the number of cascade gamma-quanta $N_{\gamma}$ arises from two reasons: resonance behavior of the cross section of $p-\gamma_{r e l}-$ interaction and the initial CR spectrum. The emitted proton interacts with the microwave background radiation until its energy decreases to 4 . $10^{19} \mathrm{eV}$. Then its free path increases to several hundred $\mathrm{Mpc}$ and the proton has a poor chance of interacting with background emission along the rest of its way to the Earth. As a result a proton from sources with a monoenergetic energy spectrum, the initial energy being $10^{21} \mathrm{eV}$, interacts with the cosmic microwave background about 10 times, initiating approximately 10 electromagnetic cascades. In sources with a CR initial exponential energy spectra the majority of protons are emitted at energies about $5 \cdot 10^{19} \mathrm{eV}$ regardless of the exponential index of the spectrum. Having long free paths, protons interact with background emission 1-2 times only, thus giving rise to 1-2 cascades. In addition, distances from near sources are too short for cascade development. The proton mean path as a function of the proton energy is shown in Fig. 1.

The quantum mean path in PP as a function of quantum energy is shown in Fig. 2, and the electron mean path in ICS versus electron energy is shown in Fig. 3.

Paths do not exceed $10 \mathrm{Mpc}$ for all quantum/electron energies. Quantum/electron propagation has no influence on the variety in the number, $N_{\gamma}$, in the models considered.

\section{Conclusion}

We estimated the intensity of gamma-ray emission at the energy $E_{\gamma}=0.1 \mathrm{TeV}$, produced by $\mathrm{CR}$ protons propagating in extragalactic space.

We analyzed three types of possible UHECR sources: remote and powerful sources located at hundreds Mpc with 


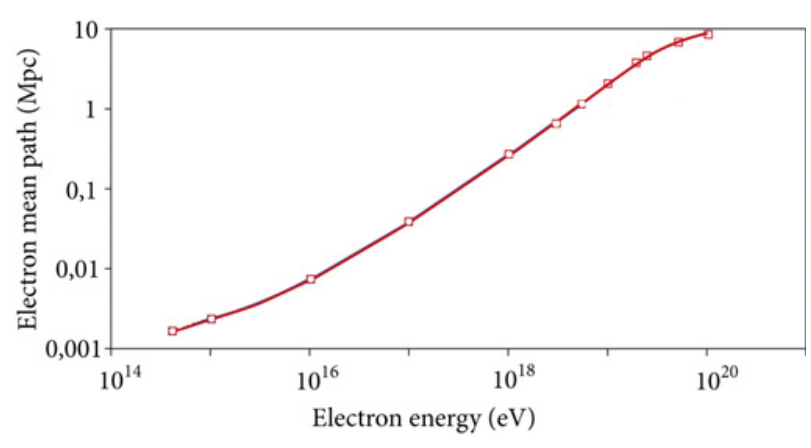

Figure 3. Electron mean path vs. its energy in ICS in extragalactic space.

red shifts up to $z=1.1$, having an initial monoenergetic spectrum, $E=10^{21} \mathrm{eV}$; remote sources but with an initial exponential energy spectra, and finally near sources located in the radius $50 \mathrm{Mpc}$, with red shifts $0<$ $z \leq 0.0092$, also having exponential spectra. Source parameters remoteness and initial energy spectrum - were chosen following [19].

We found that the cascade gamma-ray contribution in extragalactic background emission at energy $E_{\gamma}=$ $0.1 \mathrm{TeV}$ ranges from $f \ll 10^{-4}$ to $f \approx 0.1$ depending on the CR source parameters. In the estimation we used the Fermi LAT data [23].

\section{References}

[1] K. Greisen, Phys. Rev. Lett. 16, 748 (1966)

[2] G.T. Zatsepin, V.A. Kuzmin, JETP. Lett. 4, 78 (1966)
[3] M.V. Medvedev, Phys. Rev. E67, 045401 (2003)

[4] A.V. Uryson, Astron. Lett. 30, 816 (2004)

[5] S. Hayakawa, Prog. Theor. Phys. 37, 594 (1966)

[6] O. Prilutsky, I. L. Rozental, Acta Phys. Hung. Suppl. 1, 29, 51 (1970)

[7] E. Dwek, F. Krennrich, Astroparticle Physics 43, 112 (2013)

[8] Particle Data Group, Phys. Rev. D 69, 269 (2004)

[9] F. W. Stecker, Phys. Rev. Lett. 21, 1016 (1968)

[10] V.S. Berezinsky, Physics of Atomic Nuclei 11, 399 (1970)

[11] V.L. Ginzburg, Theoretical Physics and Astrophysics (Oxford, Pergamon Press, 1979)

[12] A.V. Uryson, In Proc. 34 ICRC, the Hague (2015)

[13] T. Abu-Zayyad et al., PAO and TA: Joint Contributions. In Proc. 33 ICRC, Beijing (2013)

[14] N. S. Kardashev, MNRAS 522, 205 (1995)

[15] A.V. Uryson, JETP Letters 64, 71 (1996)

[16] A.V. Uryson, Astron. Rep. 78, 686 (2001)

[17] D.S. Gorbunov et. al., ApJ 577, L93 (2002)

[18] M.P. Veron-Cetty, P. Veron, Astron. Astrophys. 412, 399 (2003)

[19] A.V. Uryson, Physics of Particles and Nuclei. 37, 347 (2006)

[20] T. A. Clark, L.W. Brown, J. K. Alexander, Nature 228, 847 (1970)

[21] R. J. Protheroe, P. L. Biermann, Astropart. Phys. 6, 45 (1996); 7, 181(E) (1997)

[22] V.S. Berezinsky et al. Ed: V.L. Ginzburg, Astrophysics of Cosmic Rays (North-Holland,1990)

[23] M. Ackermann, M. Ajello, A. Albert et al. ApJ 799, 1 (2015) 